\title{
Generalized Helly spaces, continuity of monotone functions, and metrizing maps
}

\author{
by \\ Lech Drewnowski and Artur Michalak (Poznań)
}

\begin{abstract}
Given an ordered metric space (in particular, a Banach lattice) $E$, the generalized Helly space $H(E)$ is the set of all increasing functions from the interval $[0,1]$ to $E$ considered with the topology of pointwise convergence, and $E$ is said to have property $(\lambda)$ if each of these functions has only countably many points of discontinuity. The main objective of the paper is to study those ordered metric spaces $C(K, E)$, where $K$ is a compact space, that have property $(\lambda)$. In doing so, the guiding idea comes from the fact that there is a natural one-to-one correspondence between increasing functions $f:[0,1] \rightarrow C(K, E)$ (with countably many discontinuities) and continuous maps $F: K \rightarrow H(E)$ (with metrizable ranges). It leads to the investigation of general continuous metrizing maps (those with metrizable ranges), and especially of the so called separately metrizing maps, and the results obtained are then used to derive some permanence properties of the class of spaces $C(K, E)$ with property $(\lambda)$. For instance, it is shown that if $K$ is the product of compact spaces $K_{j}(j \in J)$ such that each of the spaces $C\left(K_{j}, E\right)$ has property $(\lambda)$, so does $C(K, E)$; and, for any compact space $K$, if both $C(K)$ and a Banach lattice $E$ have property $(\lambda)$, so does $C(K, E)$.
\end{abstract}

1. Introduction. Let $E$ be a real Banach lattice. It was shown by Lavrič [8] that if $E$ is separable or if $E$ is $\sigma$-Dedekind complete and contains no lattice copy of $l_{\infty}$ (or, equivalently, $E$ has an order continuous norm), then every monotone function $f:[0,1] \rightarrow E$ has at most countably many points of discontinuity.

This direction of research was continued by the first named author in [2], where the Banach lattices satisfying the assertion of Lavrič's results were said to have property $(\lambda)$. Most of paper [2] was devoted to the question which of the Banach lattices $C(K, E)$, where $K$ is a compact space, have

2000 Mathematics Subject Classification: Primary 54E35, 54F05, 54D30, 54C05, 54B10; Secondary 46B42, 46E15.

Key words and phrases: ordered metric space, increasing function, points of discontinuity, generalized Helly space, metrizing map, separately metrizing map.

This research was partially supported by Komitet Badań Naukowych (State Committee for Scientific Research, Poland), Grant no. 2 P03A 05115. 
property $(\lambda)$. Of course, a necessary condition for this to happen is that both the spaces $C(K) \equiv C(K, \mathbb{R})$ and $E$ have property $(\lambda)$. Now, under the assumption that $E$ has property $(\lambda)$, it was proved in [2] that $C(K, E)$ has property $(\lambda)$ when

(a) each separable subspace of $K$ is metrizable, or

(b) $K$ is the product of a family of compact spaces such that $C\left(K^{\prime}, E\right)$ has property $(\lambda)$ for each finite subproduct $K^{\prime}$ of the family.

A number of open problems were posed in [2], among them the following ones:

$\left(\mathrm{a}^{\prime}\right)$ Is it true that if both $C(K)$ and $E$ have property $(\lambda)$, so does $C(K, E)$ ?

$\left(\mathrm{b}^{\prime}\right)$ Is it true that if $K_{1}$ and $K_{2}$ are compact spaces, and both $C\left(K_{1}, E\right)$ and $C\left(K_{2}, E\right)$ have property $(\lambda)$, so does $C\left(K_{1} \times K_{2}, E\right)$ ?

Note that a positive answer to question $\left(\mathrm{b}^{\prime}\right)$ would make the extra hypothesis in result (b) redundant; however, the answer to $\left(\mathrm{b}^{\prime}\right)$ was unknown even for the case $E=\mathbb{R}$.

Somewhat later, the second named author carried out in [9] a thorough investigation of the structure of those real Banach spaces $X$ endowed with the order relation induced by a norming subset of the dual space $X^{*}$ that admit an increasing function $f:[0,1] \rightarrow X$ with uncountably many discontinuities. (In isomorphic terms, he dealt with closed subspaces $X$ of $C(K)$ spaces considered with their natural pointwise order.) He proved, among other things, that a Banach lattice, in particular a $C(K)$ space, has property $(\lambda)$ iff it contains no order-isomorphic copy of the space $D(0,1)$ ( $[9$, Cor. 5]; see [9] and [10] for more information about $D(0,1))$. This was highly surprising because $D(0,1)$ entered the scene in [2, Prop. 4.2] merely as an example of a Banach lattice containing no isomorphic copy of $l_{\infty}$ and yet failing property $(\lambda)$. However, an intrinsic characterization of those compact spaces $K$ for which $C(K)$ has property $(\lambda)$ is as yet unknown.

In the present paper we answer the above questions in the affirmative. Two crucial observations that made this possible concern increasing functions $f:[0,1] \rightarrow C(K)$ with $0 \leq f \leq 1$ (a condition one can always assume without loss of generality), and have already been successfully exploited in [9]. They are the following (cf. [9, Th. 1]):

- First, there is a natural one-to-one correspondence between such functions $f$ and continuous maps $F: K \rightarrow H$, where $H$ is the Helly space, given by the equality $F(t)(s)=f(s)(t)$. (Recall that $H$ is the compact space consisting of all increasing functions from $[0,1]$ into itself and considered with the topology of pointwise convergence.) 
- Second, such a function $f$ has only countably many discontinuities iff the associated map $F$ has a metrizable range.

Thus, question $\left(\mathrm{b}^{\prime}\right)$ for the case $E=\mathbb{R}$ can be reformulated in purely topological terms as follows:

$\left(\mathrm{b}^{\prime \prime}\right)$ Suppose every continuous map from compact spaces $K_{1}$ and $K_{2}$ to $H$ has a metrizable range; is then the same true of continuous maps from $K_{1} \times K_{2}$ to $H$ ?

It turns out to be easier to deal with, and answer (in the affirmative), question $\left(b^{\prime \prime}\right)$ rather than question $\left(b^{\prime}\right)$. Moreover, from the positive answer to question $\left(\mathrm{b}^{\prime}\right)$ for the case $E=\mathbb{R}$ we are able to deduce a positive answer to question $\left(\mathrm{a}^{\prime}\right)$ in its general form. From this a positive answer to the general form of question $\left(b^{\prime}\right)$ follows immediately.

As explicitly noted in [2], most of the results proved in that paper, in particular the results (a) and (b) remain valid when $E$ is an arbitrary ordered metric space, and the questions raised therein, in particular questions $\left(\mathrm{a}^{\prime}\right)$ and $\left(b^{\prime}\right)$, make sense in this more general setting as well. However, in this case we cannot reduce our problems to $C(K)$ spaces and continuous maps $F: K \rightarrow H$. On the contrary, we have to deal directly with the spaces $C(K, E)$ and continuous maps $F: K \rightarrow H(E)$, where $H(E)$ is a natural analogue of $H$ consisting of all increasing functions from $[0,1]$ to $E$.

Motivated by what was said above, in this paper:

1) we extend some of the results known for the Helly space $H$ to the generalized Helly spaces $H(E)$, where $E$ is an ordered metric space;

2) we investigate continuous maps with metrizable ranges; we call such maps metrizing, and focus our attention on the question when a separately metrizing continuous map defined on the product of a family of compact spaces is (globally) metrizing;

3) we apply the results obtained to more general versions of questions $\left(a^{\prime}\right)$ and $\left(b^{\prime}\right)$.

2. A few preliminary facts. Throughout the paper, $I$ stands for the interval $[0,1]$; all topological spaces occurring are assumed to be Hausdorff; and, given a set $S$, we denote by $\mathcal{F}(S)$ the family of all its finite subsets.

For a function $f$ from $I$ to a topological space $X$, we denote by $D(f)$ the set of points of discontinuity of $f$. Moreover, given a set $F$ of such functions, we write $D(F)$ for the union of the sets $D(f)$, where $f \in F$.

If $X$ is a metric space, then $D(f)$ is an $F_{\sigma}$ subset of $I$ (see $[6, \S 21 . \mathrm{III}]$ ); consequently, either $|D(f)| \leq \aleph_{0}$ or $|D(f)|=2^{\aleph_{0}}$ (by [6, §37.I, Thm. 3]).

A function $f: I \rightarrow X$, where $X$ is a (partially) ordered space, is monotone if it is increasing or decreasing (in the weak sense); that is, in an 
alternative terminology, nondecreasing or nonincreasing, respectively. Since such a function $f$ is decreasing iff the function $s \mapsto f(1-s)$ is increasing, in what follows we restrict our attention to increasing functions only.

By an ordered metric space we understand a metric space $X=(X, d)$ with a (partial) order relation $\leq$ such that the metric $d$ is monotone relative to $\leq$. The latter means that whenever $x \leq u \leq v \leq y$, then $d(u, v) \leq d(x, y)$. We shall sometimes assume that the relation $\leq$ is closed as a subset of the product space $X \times X$, that is

(m) whenever $\left(x_{n}\right)$ and $\left(y_{n}\right)$ are sequences in $X$ with $x_{n} \leq y_{n}$ for all $n$, and $x_{n} \rightarrow x, y_{n} \rightarrow y$ in $X$, then $x \leq y$.

In general, for a function $f$ from the interval $I$ to a topological space, if $|D(f)| \leq \aleph_{0}$, then $f$ has a separable range. (For if $Q$ is any countable dense subset of $I$ containing $D(f) \cup\{0,1\}$, then $f(Q)$ is dense in $f(I)$.) For monotone functions taking values in ordered metric spaces, also the converse is true (see [8] and [2, Fact 1.7]); this is included in the following.

Proposition 2.1. Let $X$ be an ordered metric space, and $f: I \rightarrow X$ an increasing function. Then the following are equivalent.

(a) $|D(f)| \leq \aleph_{0}$.

(b) There is a countable set $A \subset I$ such that at each point $s \in I \backslash A$ the function $f$ satisfies the Cauchy condition for the existence of the left-hand limit $f(s-)$ or the right-hand limit $f(s+)$.

(c) The range of $f$ is separable.

Proof. That (a) implies (b) is trivial.

(b) $\Rightarrow(\mathrm{c})$ : Suppose $f(I)$ is nonseparable. Then, for some $\varepsilon>0$, one can find an uncountable set $S \subset I$ such that $d\left(f\left(s^{\prime}\right), f\left(s^{\prime \prime}\right)\right)>\varepsilon$ for all distinct points $s^{\prime}, s^{\prime \prime} \in S$. It can also be assumed that each $s \in S$ is a two-sided accumulation point of $S$. Now, take any $s \in S$. Then to the left of $s$, as well as to the right of $s$, and at the same time arbitrarily close to $s$, one can find distinct points $s^{\prime}, s^{\prime \prime}$ with $d\left(f\left(s^{\prime}\right), f\left(s^{\prime \prime}\right)\right)>\varepsilon$. This contradicts (b).

(c) $\Rightarrow$ (a): Suppose $|D(f)|>\aleph_{0}$. Then, for some $\varepsilon>0$, one can find an uncountable set $S \subset D(f)$ such that $d\left(f\left(s^{\prime}\right), f\left(s^{\prime \prime}\right)\right)>\varepsilon$ whenever $s^{\prime}<$ $s<s^{\prime \prime}$ and $s \in S$. It can also be assumed that each $s \in S$ is a two-sided accumulation point of $S$. Then $d\left(f\left(s^{\prime}\right), f\left(s^{\prime \prime}\right)\right)>\varepsilon$ for any two distinct points $s^{\prime}, s^{\prime \prime} \in S$, thus contradicting (c).

As in [2], we say that an ordered metric space $X$ has property $(\lambda)$ if every increasing function $f: I \rightarrow X$ has at most countably many points of discontinuity, i.e., $|D(f)| \leq \aleph_{0}$.

Lemma 2.2. Let $R$ be an infinite countable subset of an interval $[a, b] \subset$ $\mathbb{R}$ such that $R$ is order dense in itself (i.e., $(s, t) \cap R \neq \emptyset$ whenever $s, t \in R$ 
and $s<t$ ) and has neither a smallest nor largest element. Also, let $Q$ be a countable dense subset of $(0,1)$. Then there exists a strictly increasing function $\varphi: I \rightarrow[a, b]$ such that $\varphi(0)=a, \varphi(1)=b$, and $\varphi(Q)=R$.

Proof. It is a well known fact (see [7, Ch. VI, §3, Thm. 2]) that the assumptions on $R$ and $Q$ guarantee that there exists a strictly increasing function (an order isomorphism) $\psi$ mapping $Q$ onto $R$. We extend $\psi$ to a function $\varphi$ from $I$ into $[a, b]$ by setting $\varphi(0)=a, \varphi(1)=b$, and $\varphi(t)=$ $\sup \{\psi(s): s \in Q \cap(0, t]\}$ for $t \in(0,1)$. It is then easily seen that $\varphi$ has the desired properties.

Corollary 2.3. Let $X$ be an ordered metric space, and $f: I \rightarrow X$ an increasing function. If $|D(f)|>\aleph_{0}$, then there exists a strictly increasing function $\varphi: I \rightarrow I$ such that for the increasing function $g:=f \circ \varphi: I \rightarrow X$ one has

$$
\inf \left\{d\left(g(s), g\left(s^{\prime}\right)\right): s, s^{\prime} \in I, s \neq s^{\prime}\right\}>0 .
$$

In particular, $D(g)=I$.

Proof. By [2, Lemma 3.1], for some $\varepsilon>0$ we can find an uncountable set $D \subset I$ such that $d\left(f(s), f\left(s^{\prime}\right)\right)>\varepsilon$ for all distinct $s, s^{\prime} \in D$. It can be assumed, additionally, that each point in $D$ is a two-sided accumulation point of $D$, and that $D$ has neither a smallest nor largest element. Let $R$ be any countable dense subset of $D$, and let $Q$ be the set of rationals in $(0,1)$. By Lemma 2.2, there exists a strictly increasing function $\varphi: I \rightarrow I$ such that $\varphi(Q)=R$.

Consider the function $g:=f \circ \varphi$. Take any $t, t^{\prime} \in I$ with $t<t^{\prime}$, and next choose $s, s^{\prime} \in Q$ so that $t<s<s^{\prime}<t^{\prime}$. Then $d\left(g\left(t^{\prime}\right), g(t)\right) \geq d\left(g\left(s^{\prime}\right), g(s)\right)>$ $\varepsilon$, and the proof is complete.

Corollary 2.4. An ordered metric space $X$ fails to have property $(\lambda)$ if and only if it contains a metrically discrete order-isomorphic copy of the interval $I$, that is, there exists an increasing function $g: I \rightarrow X$ such that, for some $\varepsilon>0$, one has $d\left(g\left(t^{\prime}\right), g(t)\right)>\varepsilon$ whenever $t, t^{\prime} \in I$ and $t \neq t^{\prime}$.

We are now in a position to give an affirmative answer to Problem 1 in [2] by showing that the apparently weaker property $\left(\lambda_{0}\right)$ stated in condition (b) below implies property $(\lambda)$.

Proposition 2.5. For an ordered metric space $X$, the following are equivalent.

(a) $X$ has property $(\lambda)$.

(b) $X$ has property $\left(\lambda_{0}\right)$, that is, every increasing function $f: I \rightarrow X$ has a point of continuity in $I$.

(c) For every increasing function $f: I \rightarrow X$ and every $\varepsilon>0$ there exists a pair of distinct points $s, s^{\prime} \in I$ with $d\left(f(s), f\left(s^{\prime}\right)\right)<\varepsilon$. 
Proof. The implications (a) $\Rightarrow(\mathrm{b}) \Rightarrow(\mathrm{c})$ are trivial, and that (c) implies (a) follows directly from Corollary 2.4.

3. Spaces $C(K, E)$ with property $(\lambda)$. Preliminary facts. Let $X$ be a (Hausdorff) topological space and $E=(E, \leq, d)$ an ordered metric space. Then $C(X, E)$ stands for the set of all continuous functions $x: X \rightarrow E$ equipped with the pointwise order induced from $E$. It will be considered either with the topology $\tau_{p}$ of pointwise convergence on $X$, or with the (metrizable) topology $\tau_{u}$ of uniform convergence on $X$, and the resulting topological spaces will be indicated by $C_{p}(X, E)$ and $C_{u}(X, E)$, respectively. Clearly, if $E$ satisfies condition (m), so does $C_{p}(X, E)$ and a fortiori $C_{u}(X, E)$. For each point $t \in X$ we denote by $p_{t}$ the evaluation map on $C(X, E)$ defined by $p_{t}(x)=x(t)$.

Given a function $f: I \rightarrow C(X, E)$, it will often be convenient to write $f(s, t)$ instead of $f(s)(t)$ for $s \in I$ and $t \in X$. Clearly, $f(\cdot, t)=p_{t} \circ f$ for each $t \in X$. Evidently, if $|D(f)| \leq \aleph_{0}$, then $\left|D\left(p_{t} \circ f\right)\right| \leq \aleph_{0}$ for each $t \in X$.

In most cases occurring below, the domain space $X$ is assumed to be compact and, as a rule, we denote it by $K$. Note that in this case the topology $\tau_{u}$ is defined by the standard supremum (extended) metric $d_{\infty}$, and we usually write simply $C(K, E)$ instead of $C_{u}(K, E)$ or $\left(C(K, E), d_{\infty}\right)$.

We are interested in continuity type properties of increasing functions $f: I \rightarrow C(K, E)$. In particular, we would like to know under what circumstances the space $C(K, E)$ has property $(\lambda)$; an obvious necessary condition is that $E$ has property $(\lambda)$. We start with the following observation.

Proposition 3.1. Let $K$ be a compact space. Then an increasing function $f: I \rightarrow C(K, E)$ is continuous at a point $s_{0} \in I$ (for the metric topology of $\left.d_{\infty}\right)$, that is,

$$
\lim _{s \rightarrow s_{0}} d_{\infty}\left(f(s), f\left(s_{0}\right)\right)=0,
$$

if and only if it is continuous at $s_{0}$ for the topology $\tau_{p}$, that is,

$$
\lim _{s \rightarrow s_{0}} d\left(f(s, t), f\left(s_{0}, t\right)\right)=0 \quad \forall t \in K ;
$$

in other words, each of the increasing functions $p_{t} \circ f: I \rightarrow E(t \in K)$ is continuous at $s_{0}$. Likewise for the left-and right-continuity at $s_{0}$.

Consequently,

$$
D(f)=\bigcup_{t \in K} D\left(p_{t} \circ f\right)=D\left(\left\{p_{t} \circ f: t \in K\right\}\right) .
$$

Proof. The "if" part: Given any monotone sequence $\left(s_{n}\right)$ in $I$ converging to $s_{0}$, the sequence $d\left(f\left(s_{n}, \cdot\right), f\left(s_{0}, \cdot\right)\right)$ of continuous real-valued functions on $K$ is decreasing and converges pointwise to the zero function. Apply the Dini theorem (see e.g. [4, 3.2.18]). 
Proposition 3.2. Let $X$ be a topological space, and let $f: I \rightarrow C(X, E)$ be an increasing function. Then the set $X_{0}$ of all points $t \in X$ with $|D(f(\cdot, t))|$ $\leq \aleph_{0}$ is sequentially closed.

Proof. Let a sequence $\left(t_{n}\right)$ in $X_{0}$ converge to a point $t_{0} \in X$. Then the functions $f\left(\cdot, t_{n}\right)$ converge to $f\left(\cdot, t_{0}\right)$ pointwise on $I$, and their ranges $f\left(I, t_{n}\right)$ are all separable. It follows that also $f\left(I, t_{0}\right)$ is separable, as a subset of the closure of the union of the $f\left(I, t_{n}\right)$ 's. Hence $\left|D\left(f\left(\cdot, t_{0}\right)\right)\right| \leq \aleph_{0}$ by Proposition 2.1.

COROllary 3.3. Let $X$ be a first countable topological space. Then for every increasing function $f: I \rightarrow C(X, E)$ the set of all points $t \in X$ with $|D(f(\cdot, t))| \leq \aleph_{0}$ is closed.

Of the three results stated below, the first is a strengthened version of Theorem 5.1 in [2], with an alternative proof. The second is an extraction from the proof of Theorem 5.13 in [2], and from it the third result is derived as a corollary.

TheOREM 3.4. Let $K$ be a metrizable compact space. If $f: I \rightarrow C(K, E)$ is an increasing function such that $\left|D\left(p_{t} \circ f\right)\right| \leq \aleph_{0}$ for a dense set of points $t \in K$, then also $|D(f)| \leq \aleph_{0}$. Consequently, if $E$ has property $(\lambda)$, so does $C(K, E)$.

Proof. By Corollary 3.3 it is immediate that $\left|D\left(p_{t} \circ f\right)\right| \leq \aleph_{0}$ for every $t \in K$. From this, repeating almost verbatim the proof of Theorem 5.1 in [2], we get the desired conclusion.

Alternatively, from the assumptions and Proposition 2.1 it follows that there is a dense countable subset $C$ of $K$ such that $f(I, t)$ is separable for each point $t \in C$. Let $F$ denote the closure in $E$ of the union of the sets $f(I, t), t \in C$. Then $F$ is a separable subspace of $E$, and $f(I, t) \subset F$ for every $t \in K$ (cf. the proof of Proposition 3.2). Consequently, we may view $f$ as a function with values in the ordered metric space $C(K, F)$ which, in addition, is separable. To finish, appeal to Proposition 2.1.

Note. For the separability of $C(K, F)$, see $[6, \S 22 . \mathrm{III}]$ or $[4,4.2 .18]$. It can also be shown as follows: By the Banach-Mazur theorem (see [1, Ch. XI, Th. 10]), $F$ is isometric to a subset of the Banach space $C(I)$. Hence, in turn, $C(K, F)$ is isometric to a subset of the Banach space $C(K, C(I)) \cong C(K \times I)$, and the latter space is separable (see [13, 7.6.2]) because $K \times I$ is a metrizable compact space.

Proposition 3.5. Let $K$ be a compact space, and $f: I \rightarrow C(K, E)$ an increasing function. Then there exists a separable compact subspace $K_{0}$ of $K$ such that the increasing function $g: s \mapsto f(s) \mid K_{0}$ from $I$ to $C\left(K_{0}, E\right)$ 
has the same set of discontinuities as $f: D(g)=D(f)$. Consequently, if $|D(f)|>\aleph_{0}$, then also $|D(g)|>\aleph_{0}$.

Proof. Let $Q$ denote the set of rationals in $I$. For each pair $r, r^{\prime}$ of distinct points in $Q$ choose a point $t_{r, r^{\prime}} \in K$ so that

$$
d_{\infty}\left(f(r), f\left(r^{\prime}\right)\right)=d\left(f\left(r, t_{r, r^{\prime}}\right), f\left(r^{\prime}, t_{r, r^{\prime}}\right)\right),
$$

and let $K_{0}$ denote the closure of the set of all those points $t_{r, r^{\prime}}$.

If $s_{1} \leq s_{1}^{\prime}<s_{2}^{\prime} \leq s_{2}$ with $s_{1}^{\prime}, s_{2}^{\prime} \in Q$, then

$$
d_{\infty}\left(f\left(s_{2}\right), f\left(s_{1}\right)\right) \geq d_{\infty}\left(g\left(s_{2}\right), g\left(s_{1}\right)\right) \geq d_{\infty}\left(g\left(s_{2}^{\prime}\right), g\left(s_{1}^{\prime}\right)\right)=d_{\infty}\left(f\left(s_{2}^{\prime}\right), f\left(s_{1}^{\prime}\right)\right) .
$$

From this it follows directly that $D(f)=D(g)$.

Let us say that a compact space is separably metrizable if all its separable subspaces are metrizable.

COROLlary 3.6. If a compact space $K$ is separably metrizable, and the space $E$ has property $(\lambda)$, so does the space $C(K, E)$.

REMARK 3.7. It is obvious that closed subspaces, continuous images, and countable products of separably metrizable compact spaces are again of the same type. Note that this class of spaces includes all Corson compacts, in particular, all Eberlein compacts (see [11] for more information on Corson, Eberlein and other related classes of compact spaces). It is also worth pointing out that the compact space $W$ of all ordinal numbers $\leq \omega_{1}$ (see $[4,3.1 .27])$ is in this class but is not a Corson compact.

4. Generalized Helly spaces. The usual Helly space $H$ is the set of all increasing functions mapping the interval $I$ into itself, equipped with the topology of pointwise convergence. Its basic properties are stated in [5, Ch. 5, Problem M]: $H$ is a separable, nonmetrizable, first-countable compact Hausdorff space. Moreover, a compact subspace $K$ of $H$ is metrizable iff $|D(K)| \leq \aleph_{0}$. As shown in [12], the latter condition characterizes, in fact, those subspaces $K \subset H$ that are separable and metrizable. It was also proved there that $H$ contains a homeomorphic copy of every separable metrizable space, and that metrizable compact subsets of $H$ are $G_{\delta}$ sets.

Additionally, observe that $H$ does contain nonseparable compact subspaces. For instance, such is the subspace $K$ consisting of all functions of the form $\chi_{(r, 1]}, \chi_{[r, 1]}$, and $\frac{1}{2} \chi_{\{r\}}+\chi_{(r, 1]}$, where $r \in I$. $\left(\chi_{J}\right.$ denotes the characteristic function of a set $J \subset I$.)

It is obvious that the definition of the Helly space admits various extensions. The following one is the most suitable for our purposes.

Let $E=(E, \leq, d)$ be an ordered metric space.

By the Helly space determined by $E$ we shall mean the set $H(E) \equiv$ $H(I, E)$ of all increasing functions $x: I \rightarrow E$ equipped with the topol- 
ogy $\tau_{p}$ of pointwise convergence on $I$. Thus for each $x \in H(E)$ a base of $\tau_{p}$-neighborhoods of $x$ is formed by the sets

$$
U(x ; A, \varepsilon):=\{y \in H(E): d(y(s), x(s))<\varepsilon \forall s \in A\},
$$

where $A \in \mathcal{F}(I)$ and $\varepsilon>0$.

Given $x \in H(E)$ and a subset $S$ of $I$, we define

$$
\mathcal{U}(x ; S)=\{U(x ; A, \varepsilon): A \in \mathcal{F}(S), \varepsilon>0\} .
$$

Proposition 4.1. If the space $E$ satisfies condition $(\mathrm{m})$, then $H(E)$ is a closed subspace of the space $E^{I}$ of all functions from $I$ to $E$.

Most of our results will be concerned with the following subspace of $H(E)$ :

$$
H_{0}(E):=\left\{x \in H(E):|D(x)| \leq \aleph_{0}\right\} .
$$

Obviously, $H(E)=H_{0}(E)$ iff $E$ has property $(\lambda)$.

Proposition 4.2. $H_{0}(E)$ is sequentially closed in $H(E)$.

Proof. Define $f: I \rightarrow C_{p}(H(E), E)$ by $f(s)(x)=x(s)$. Then $f$ is increasing, and $H_{0}(E)$ coincides with the set of points $x \in H(E)$ with $|D(f(\cdot, x))| \leq \aleph_{0}$. To finish, apply Proposition 3.2.

We will need the following two lemmas.

Lemma 4.3. For every $\varepsilon>0$ there is $\delta>0$ such that if $x, y, z, x^{\prime}, z^{\prime} \in E$, $x^{\prime} \leq z^{\prime}$, and

$$
d(y, x)<\delta, \quad d(y, z)<\delta, \quad d\left(x^{\prime}, x\right)<\delta, \quad d\left(z^{\prime}, z\right)<\delta,
$$

then $d\left(y^{\prime}, y\right)<\varepsilon$ for each point $y^{\prime}$ in the order interval $\left[x^{\prime}, z^{\prime}\right]$.

Proof. Let $y^{\prime} \in\left[x^{\prime}, z^{\prime}\right]$. Then, using the monotonicity of $d$ to justify the second inequality below, we have

$$
\begin{aligned}
d\left(y^{\prime}, y\right) & \leq d\left(y^{\prime}, x^{\prime}\right)+d\left(x^{\prime}, x\right)+d(x, y) \leq d\left(z^{\prime}, x^{\prime}\right)+d\left(x^{\prime}, x\right)+d(x, y) \\
& \leq d\left(z^{\prime}, z\right)+d(z, y)+d(y, x)+d\left(x, x^{\prime}\right)+d\left(x^{\prime}, x\right)+d(x, y) \\
& =2 d(y, x)+d(y, z)+2 d\left(x^{\prime}, x\right)+d\left(z^{\prime}, z\right),
\end{aligned}
$$

which is $<\varepsilon$ provided that $\delta<\varepsilon / 6$.

Lemma 4.4. Let $x \in H(E)$ and let $Q$ be a dense subset of I containing $D(x) \cup\{0,1\}$. If $y \in H(E)$ and $x|Q=y| Q$, then $x=y$.

Proof. Let $s \in I \backslash Q$. If $s^{\prime}, s^{\prime \prime} \in Q$ and $s^{\prime}<s<s^{\prime \prime}$, then $y\left(s^{\prime}\right)=x\left(s^{\prime}\right) \leq$ $x(s), y(s) \leq x\left(s^{\prime \prime}\right)=y\left(s^{\prime \prime}\right)$, hence

$$
d(y(s), x(s)) \leq d\left(y(s), x\left(s^{\prime}\right)\right)+d\left(x\left(s^{\prime}\right), x(s)\right) \leq 2 d\left(x\left(s^{\prime \prime}\right), x\left(s^{\prime}\right)\right) .
$$

Since $x$ is continuous at $s$, it follows that $d(x(s), y(s))=0$. 
Proposition 4.5. Let $x \in H(E)$, and let $Q$ be a dense subset of the interval $I$ containing its endpoints. Then the following are equivalent.

(a) $Q \supset D(x)$.

(b) The family $\mathcal{U}(x ; Q)$ is a base of neighborhoods of $x$ in the space $H(E)$.

Proof. (a) $\Rightarrow(\mathrm{b})$ : Consider a "typical" neighborhood $U(x ; A, \varepsilon)$ of $x$ in $H(E)$, where $A \in \mathcal{F}(I)$ and $\varepsilon>0$. We are going to show that it contains a member of $\mathcal{U}(x ; Q)$. This is trivial when $A \subset Q$, so assume that $A \not \subset Q$.

Let $0<\delta<\varepsilon$ be as required in Lemma 4.3. For each $s \in B:=A \backslash Q$ choose $s^{\prime}, s^{\prime \prime} \in Q$ so that $s^{\prime}<s<s^{\prime \prime}$ and

(1) $d\left(x\left(s^{\prime}\right), x(s)\right)<\delta, d\left(x\left(s^{\prime \prime}\right), x(s)\right)<\delta$;

this is possible because $x$ is continuous at $s$. Denote by $B^{\prime}$ the set formed by all the points $s^{\prime}$ and $s^{\prime \prime}$ thus selected. Clearly, $A^{\prime}:=B^{\prime} \cup(A \cap Q)$ is a finite subset of $Q$. Now, let $y \in U\left(x ; A^{\prime}, \delta\right)$; thus

(2) $d(y(t), x(t))<\delta$ for each $t \in A^{\prime}$.

Take any $s \in A$. If $s \in A \cap Q$, then we obviously have $d(y(s), x(s))<\varepsilon$. If $s \in B=A \backslash(A \cap Q)$, then $y\left(s^{\prime}\right) \leq y(s) \leq y\left(s^{\prime \prime}\right)$, and in view of conditions (1) and (2) Lemma 4.3 gives $d(y(s), x(s))<\varepsilon$. Thus $U\left(x ; A^{\prime}, \delta\right) \in \mathcal{U}(x ; Q)$ and $U\left(x ; A^{\prime}, \delta\right) \subset U(x ; A, \varepsilon)$.

(b) $\Rightarrow(\mathrm{a})$ : Suppose there is a point $t \in D(x) \backslash Q$. Then $0<t<1$ and for some $\varepsilon>0$ we have either

(i) $d(x(s), x(t))>\varepsilon$ for all $s \in[0, t)$, or

(ii) $d(x(s), x(t))>\varepsilon$ for all $s \in(t, 1]$.

We are going to show that the neighborhood $U(x ;\{t\}, \varepsilon)$ of $x$ contains no neighborhood from $\mathcal{U}(x ; Q)$.

Suppose, for instance, that (i) holds, and let $A$ be a finite nonempty subset of $Q$. Define $a=\min A$. If $a<t$, then let $c=\max (A \cap[0, t))$ and define a function $y \in H(E)$ by setting $y(s)=x(s)$ for $s \in[0, c) \cup(t, 1]$ and $y(s)=x(c)$ for $s \in[c, t]$. If $a>t$, then define a function $y \in H(E)$ by $y(s)=x(0)$ for $s \in[0, t]$ and $y(s)=x(s)$ for $s \in(t, 1]$. In either case we have $y(s)=x(s)$ for all $s \in A$ so that $y \in U(x ; A, \delta) \in \mathcal{U}(x ; Q)$ for every $\delta>0$. However, $d(y(t), x(t))>\varepsilon$ and thus $y \notin U(x ;\{t\}, \varepsilon)$.

Corollary 4.6. A point $x \in H(E)$ has a countable base of neighborhoods iff $|D(x)| \leq \aleph_{0}$. Consequently, $H_{0}(E)$ is precisely the subspace of $H(E)$ formed by the points that have a countable base of neighborhoods in $H(E)$.

Proof. The "if" part is immediate from Proposition 4.5 by taking for $Q$ any countable dense subset of $I$ containing $D(x) \cup\{0,1\}$. 
"Only if": Suppose $x$ has a countable base of neighborhoods. Clearly, we may assume that it is formed by the sets $U\left(x ; A, n^{-1}\right)$, where $A$ is any finite subset of a certain countable dense set $Q \subset I$, and $n \in \mathbb{N}$. By Proposition 4.5, $D(x) \subset Q \cup\{0,1\}$, hence $|D(x)| \leq \aleph_{0}$.

For a subset $A$ of $H(E)$ and any $s \in I$, we define

$$
A(s)=\{x(s): x \in A\}, \quad R(A)=\bigcup_{x \in A} x(I), \quad D(A)=\bigcup_{x \in A} D(x) .
$$

Proposition 4.7. If $F$ is a separable subspace of $E$, then $H(F)=$ $H_{0}(F)$ is a separable subspace of $H_{0}(E)$.

Proof. The inclusion $H(F) \subset H_{0}(E)$ follows from Proposition 2.1. It remains to verify that $H(F)$ is separable.

First of all note that for each $n \in \mathbb{N}$ the product space $F^{n}$ is metrizable and separable, hence so is its subspace

$$
\left(F^{n}\right)_{\uparrow}:=\left\{\left(v_{1}, \ldots, v_{n}\right) \in F^{n}: v_{1} \leq \cdots \leq v_{n}\right\} .
$$

Let $M_{n}$ be a countable dense subset of $\left(F^{n}\right)_{\uparrow}$. Next, denote by $Z_{n}$ the countable set of all functions $z: I \rightarrow E$ such that for a sequence of rationals $0=r_{0}<r_{1}<\cdots<r_{n}=1$ and a sequence $\left(u_{1}, \ldots, u_{n}\right) \in M_{n}$ we have $z(s)=u_{i}$ for $s \in\left[r_{i-1}, r_{i}\right)(1 \leq i \leq n-1)$, and $z(s)=u_{n}$ for $s \in\left[r_{n-1}, r_{n}\right]$. Let $Z$ denote the union of the $Z_{n}$ 's; clearly, $Z \subset H(F)$.

We now show that $Z$ is dense in $H(F)$. Let $x \in H(F)$, take any points $s_{1}<\cdots<s_{n}$ in $I$, and fix any number $\varepsilon>0$. Next, choose rationals $0=r_{0}<r_{1}<\cdots<r_{n}=1$ so that $s_{i} \in\left[r_{i-1}, r_{i}\right)$ for $i=1, \ldots, n-1$, and $s_{n} \in\left[r_{n-1}, r_{n}\right]$. Since $\left(x\left(s_{1}\right), \ldots, x\left(s_{n}\right)\right) \in\left(F^{n}\right)_{\uparrow}$, we can find $\left(u_{1}, \ldots, u_{n}\right) \in M_{n}$ so that $d\left(x\left(s_{i}\right), u_{i}\right)<\varepsilon$ for $i=1, \ldots, n$. Finally, define a function $z: I \rightarrow E$ as in the preceding paragraph. Then $z \in Z_{n} \subset Z$ and $d\left(x\left(s_{i}\right), z\left(s_{i}\right)\right)<\varepsilon$ for $i=1, \ldots, n$, which concludes the proof.

Corollary 4.8. For a subspace $A$ of $H(E)$ the following are equivalent.

(a) $A$ is contained in a separable subspace of $H_{0}(E)$.

(b) $R(A)$ is a separable subspace of $E$.

Proof. (a) $\Rightarrow(\mathrm{b})$ : Suppose $A$ is contained in a separable subspace $B$ of $H_{0}(E)$, and let $C$ be a countable dense subset of $B$. Each $x \in B$ has a separable range because $|D(x)| \leq \aleph_{0}$. It follows that the closure $S$ of $R(C)$ in $E$ is separable. To finish note that $R(A) \subset R(B) \subset S$.

(b) $\Rightarrow($ a): If $F:=R(A)$ is separable then, by Proposition 4.7, so is the subspace $H(F)$ of $H_{0}(E)$, and it obviously contains $A$.

Proposition 4.9. A compact subspace $K$ of $H_{0}(E)$ is metrizable iff $|D(K)| \leq \aleph_{0}$. 
Proof. Suppose $|D(K)| \leq \aleph_{0}$ and let $Q$ be any countable dense subset of $I$ containing $D(K) \cup\{0,1\}$. By Lemma 4.4, if $x, y \in K$ and $x|Q=y| Q$, then $x=y$. This means that the restriction map $x \mapsto x \mid Q$ from $K$ into $\prod_{s \in Q} K(s)$ is one-to-one. Moreover, it is continuous, and the factors in the product are all compact subsets in the metric space $E$. Consequently, we have a homeomorphic embedding of $K$ into a metrizable compact space, hence $K$ itself is metrizable.

Conversely, let $K$ be metrizable. Define a function $f: I \rightarrow C(K, E)$ by $f(s)(x)=x(s)$. Then $f$ is increasing, and $D(f)=D(K)$, as follows easily from equality $(*)$ in Proposition 3.1. Moreover, for each $x \in K$ one has $p_{x} \circ f=x$, and $|D(x)| \leq \aleph_{0}$ by assumption. Therefore, we may apply Theorem 3.4 to deduce that $|D(f)| \leq \aleph_{0}$.

For the usual Helly space $H$, the following has been shown in [9, Fact 2].

Corollary 4.10. Every nonmetrizable compact subspace $K$ of $H_{0}(E)$ contains another compact subspace $L$ which is nonmetrizable and separable. In other words, a separably metrizable compact subspace of $H_{0}(E)$ is metrizable.

Proof. Let $f$ be the function defined as in the proof above. Then, by Proposition 3.5, there is a separable closed subset $L$ of $K$ such that for the function $g: s \mapsto f(s) \mid L$ one has $D(K)=D(f)=D(g)=D(L)$. It follows that $|D(L)|>\aleph_{0}$ so that $L$ is nonmetrizable by the previous proposition.

It is worth noting that an inspection of the argument used in the proof of Fact 2 in [9] mentioned above reveals that, in fact, it is a proof of a much stronger result. (For scattered spaces, see [13, Sec. 8.5].)

Proposition 4.11. Every nonmetrizable compact subspace $K$ of $H$ contains another compact subspace $L$ which is nonmetrizable, nonscattered, and separable. Consequently, scattered compact subspaces of $H$ are metrizable, and therefore countable.

Proposition 4.9 is, actually, a particular case of a more general fact:

Proposition 4.12. A compact subspace $K$ of a product $X=\prod_{j \in J} X_{j}$ of metrizable spaces is metrizable iff there exists a countable subset $J_{0}$ of $J$ such that whenever $x, y \in K$ and $x\left|J_{0}=y\right| J_{0}$, then $x=y$.

Proof. The argument for the "if" part is like that in the proof of Proposition 4.9. The "only if" part follows from the fact that the identity map from $K \subset X$ to $K$ depends only on countably many coordinates $j \in J$ (see [3, p. 221], [4, 3.2.H], and [2, Lemma 5.8]).

The "if" part of Proposition 4.9 admits a more general version: 
Proposition 4.13. Let $A$ be a subspace of $H(E)$, and $Q$ a dense subset of $I$ containing $D(A) \cup\{0,1\}$. Then the restriction map $R_{Q}: x \mapsto x \mid Q$ is a homeomorphic embedding of $A$ into the product space $E^{Q}$. Consequently, if $|D(A)| \leq \aleph_{0}$, then the subspace $A$ is metrizable, and if in addition $A(s) \subset E$ is separable for each $s \in I$, then also $A$ is separable.

Proof. By Proposition 4.5, for every $x \in A$ the family $\mathcal{U}(x ; Q)$ is a base of neighborhoods of the point $x$ in $H(E)$. From this it follows that $R_{Q}$ has a continuous inverse on $R_{Q}(A)$.

Thus, in particular, every countable subspace $A$ of $H_{0}(E)$ is metrizable.

The following result is a generalization of [12, Prop. 2].

Proposition 4.14. For a subspace $A$ of $H_{0}(E)$ the following are equivalent.

(a) $A$ is metrizable and separable.

(b) $|D(A)| \leq \aleph_{0}$ and $R(A)$ is separable.

Proof. (b) $\Rightarrow($ a): This is contained in Proposition 4.13.

(a) $\Rightarrow(\mathrm{b}): F:=R(A)$ is separable by Corollary 4.8 .

Suppose $|D(A)|>\aleph_{0}$. Then with no loss of generality we may assume that the set $D_{r}(A)$ of points $t \in I$ where at least one of the functions $x \in A$ is not continuous from the right is uncountable. Next, remembering that $|D(x)| \leq \aleph_{0}$ for every $x \in A$, we can find a number $\varepsilon>0$ and an uncountable subset $D$ of $D_{r}(A)$ such that for each $s \in D$ there is a function $x_{s} \in A$ for which $d\left(x_{s}(s), x_{s}(t)\right) \geq \varepsilon$ for all $t \in(s, 1]$. We may assume that $x_{s} \neq x_{s^{\prime}}$ whenever $s \neq s^{\prime}$.

Since $F$ is separable, it can be covered by a sequence of open balls $K\left(z_{n}, r\right)(n \in \mathbb{N})$ with centers $z_{n} \in F$ and radius $r=\varepsilon / 3$. Clearly, there is an index $m$ for which the set $D_{0}:=\left\{s \in D: x_{s}(s) \in K\left(z_{m}, r\right)\right\}$ is uncountable.

Now, for each $s \in D_{0}$ the set $U_{s}:=\left\{x \in A: x(s) \in K\left(z_{m}, r\right)\right\}$ is open in $A$ and contains $x_{s}$. On the other hand, if $s, s^{\prime} \in D_{0}$ and $s<s^{\prime}$, then $x_{s} \notin U_{s^{\prime}}$. Indeed,

$$
d\left(x_{s}\left(s^{\prime}\right), z_{m}\right) \geq d\left(x_{s}\left(s^{\prime}\right), x_{s}(s)\right)-d\left(x_{s}(s), z_{m}\right) \geq \varepsilon-\frac{1}{3} \varepsilon=\frac{2}{3} \varepsilon>r .
$$

Since $A$ is separable and metrizable, its topology has a countable base, say $\mathcal{B}$. Then for each $s \in D_{0}$ there is a $B_{s} \in \mathcal{B}$ such that $x_{s} \in B_{s} \subset U_{s}$. Since $\left|D_{0}\right|>\aleph_{0}$, there must be distinct $s, s^{\prime} \in D_{0}$ with $B_{s}=B_{s^{\prime}}$. Then both $x_{s}$ and $x_{s^{\prime}}$ would belong to each of the sets $U_{s}$ and $U_{s^{\prime}}$, which, as we have just seen, is impossible.

The next two results, Proposition 4.15 and Corollary 4.18, are extensions to the spaces $H(E)$ of analogous results proved in [12, p. 44] for the usual Helly space. 
Proposition 4.15. For a compact subset $K$ of $H(E)$ the following are equivalent.

(a) There is a countable set $Q \subset I$ such that whenever $x \in K, y \in H(E)$, and $x|Q=y| Q$, then $y \in K$.

(b) $K$ is a $G_{\delta}$ subset of $H(E)$.

Proof. (a) $\Rightarrow(\mathrm{b})$ : Let $Q=\left\{s_{1}, s_{2}, \ldots\right\}$. For every $x \in K$ and $n \in \mathbb{N}$ set $U_{n}(x)=U\left(x ; Q_{n}, n^{-1}\right)$, where $Q_{n}:=\left\{s_{1}, \ldots, s_{n}\right\}$. Define

$$
G_{n}=\bigcup_{x \in K} U_{n}(x) \quad(n \in \mathbb{N}) \quad \text { and } \quad G=\bigcap_{n=1}^{\infty} G_{n} .
$$

Clearly, $K \subset G$. To finish, it suffices to verify that $G \subset K$.

Let $y \in G$. Then for each $n$ there is $x_{n} \in K$ such that $y \in U_{n}\left(x_{n}\right)$. Thus

$$
d\left(y\left(s_{i}\right), x_{n}\left(s_{i}\right)\right)<n^{-1} \quad \text { for } i=1, \ldots, n .
$$

Since $K$ is compact, the sequence $\left(x_{n}\right)$ has a cluster point $x \in H(E)$. Evidently, $y(s)=x(s)$ for every $s \in Q$. Hence $y \in K$, by (a).

An alternative argument: Consider the continuous map $f: x \mapsto x \mid Q$ from $H(E)$ into the metrizable compact space $L:=\prod_{s \in Q} K(s)$. Then $K^{\prime}:=$ $f(K)$ is a compact, hence $G_{\delta}$, subset of $L$. Moreover, by (a), whenever $y \in H(E) \backslash K$, then $f(y) \notin K^{\prime}$. This means that $K=f^{-1}\left(K^{\prime}\right)$, hence $K$ is a $G_{\delta}$ subset of $H(E)$.

(b) $\Rightarrow(\mathrm{a})$ : Let $\left(G_{n}\right)$ be a sequence of open sets in $H(E)$ such that its intersection is equal to $K$. Note that for each $x \in K$ and $n \in \mathbb{N}$ there is a neighborhood of $x$ of the type $U(x ; A, \varepsilon)(A \in \mathcal{F}(I), \varepsilon>0)$ contained in $G_{n}$. Hence, by the compactness of $K$, for every $n$ we can find points $x_{n, 1}, \ldots, x_{n, m_{n}} \in K$, sets $A_{n, 1}, \ldots, A_{n, m_{n}} \in \mathcal{F}(I)$ and positive numbers $\varepsilon_{n, 1}, \ldots, \varepsilon_{n, m_{n}}$ such that

$$
K \subset \bigcup_{i=1}^{m_{n}} U\left(x_{n, i} ; A_{n, i}, \varepsilon_{n, i}\right) \subset G_{n} .
$$

Let

$$
Q:=\bigcup_{n=1}^{\infty} \bigcup_{i=1}^{m_{n}} A_{n, i}
$$

Suppose $x \in K, y \in H(E)$, and $x|Q=y| Q$. Since $x \in K$, for each $n$ there is $i_{n}$ such that $x \in U\left(x_{n, i_{n}} ; A_{n, i_{n}}, \varepsilon_{n, i_{n}}\right)$. But $x=y$ on $A_{n, i_{n}}$, hence also $y \in U\left(x_{n, i_{n}} ; A_{n, i_{n}}, \varepsilon_{n, i_{n}}\right)$. Thus $y \in G_{n}$ for every $n$, and consequently $y \in K$.

It is worth noting that Proposition 4.15 admits a more general version:

Proposition 4.16. Let $M$ be a subspace of a product $\prod_{s \in S} X_{s}$ of metrizable spaces. Then for a compact subset $K$ of $M$ the following are equivalent. 
(a) There is a countable set $T \subset S$ such that whenever $x \in K, y \in M$, and $x|T=y| T$, then $y \in K$.

(b) $K$ is a $G_{\delta}$ subset of $M$.

Corollary 4.17. A point $x \in H(E)$ is a $G_{\delta}$-point iff there is a countable set $Q \subset I$ such that whenever $y \in H(E)$ and $x|Q=y| Q$, then $x=y$.

COROLlary 4.18. Every metrizable compact subspace $K$ of $H_{0}(E)$ is a $G_{\delta}$ subset of $H(E)$.

Proof. By Proposition 4.9, $|D(K)| \leq \aleph_{0}$. In view of Lemma 4.4, any countable dense subset $Q$ of $I$ containing $D(K) \cup\{0,1\}$ is as required in condition (a) of Proposition 4.15.

With the help of points of order-discontinuity, we are now going to give more precise versions of Lemma 4.4 and Corollary 4.17. We shall say that a function $x \in H(E)$ is order-continuous at a point $s_{0} \in I$ if

$$
x\left(s_{0}\right)=\sup \left\{x(s): s \in\left[0, s_{0}\right)\right\} \quad \text { and } \quad x\left(s_{0}\right)=\inf \left\{x(s): s \in\left(s_{0}, 1\right]\right\},
$$

with the proviso that only the first or the second equality is required to hold when $s_{0}=1$ or $s_{0}=0$, respectively.

Proposition 4.19. If $x \in H(E)$ is continuous at a point $s_{0} \in I$, then $x$ is also order-continuous at $s_{0}$.

For each $x \in H(E)$, let $D_{o}(x)$ denote the set of all points in $I$ where $x$ is not order-continuous. By the proposition above, $D_{o}(x) \subset D(x)$. In general, these two sets may be radically different. For instance, if $E$ is the unit interval $[0,1]$ with the natural order and the discrete (zero-one) metric, and $x \in H(E)$, then

(a) $D_{o}(x)$ coincides with the set of usual points of discontinuity of $x$ (i.e., those for the natural metric of $E$ ) so that $\left|D_{o}(x)\right| \leq \aleph_{0}$;

(b) $|D(x)| \leq \aleph_{0}$ iff $|x(I)| \leq \aleph_{0}$ (by Proposition 2.1).

Proposition 4.20. Let $x \in H(E)$, and let $Q$ be a dense subset of the interval I containing its endpoints. Then the following are equivalent.

(a) $Q \supset D_{o}(x)$.

(b) Whenever $y \in H(E)$ and $x|Q=y| Q$, then $x=y$.

(c) The intersection of the neighborhoods $U \in \mathcal{U}(x ; Q)$ is equal to $\{x\}$.

Proof. It is quite obvious that conditions (b) and (c) are indeed equivalent.

$(\mathrm{a}) \Rightarrow(\mathrm{b})$ : Let $y$ be as required in (b), and let $s_{0} \in I \backslash Q$. Since $x$ is order-continuous at $s_{0}$ and $Q \cap\left[0, s_{0}\right)$ is a cofinal subset of $\left[0, s_{0}\right)$, we have $x\left(s_{0}\right)=\sup \left\{x(s): s \in Q \cap\left[0, s_{0}\right)\right\}$. On the other hand, $x(s)=y(s)$ for all $s \in Q \cap\left[0, s_{0}\right)$, hence $x\left(s_{0}\right) \leq y\left(s_{0}\right)$. Likewise, $y\left(s_{0}\right) \leq x\left(s_{0}\right)$. Thus $x\left(s_{0}\right)=y\left(s_{0}\right)$. 
(b) $\Rightarrow\left(\right.$ a): Suppose there is a point $s_{0} \in D_{o}(x) \backslash Q$. Then $0<s_{0}<1$ and for some $z \in E$ we have either

(i) $x(s) \leq z<x\left(s_{0}\right)$ for all $s \in\left[0, s_{0}\right)$, or

(ii) $x\left(s_{0}\right)<z \leq x(s)$ for all $s \in\left(s_{0}, 1\right]$.

In either case, define a function $y \in H(E)$ by setting $y(s)=x(s)$ for $s \neq s_{0}$ and $y\left(s_{0}\right)=z$. Obviously, $y|Q=x| Q$ and $y \neq x$, contradicting (b).

COROllary 4.21. A point $x \in H(E)$ is a $G_{\delta}$-point iff $\left|D_{o}(x)\right| \leq \aleph_{0}$.

Proof. Suppose $\left|D_{o}(x)\right| \leq \aleph_{0}$ and take any countable dense subset $Q$ of $I$ containing $D_{o}(x) \cup\{0,1\}$. By Proposition 4.20, $\{x\}$ is equal to the intersection of the neighborhoods $U\left(x ; A, n^{-1}\right)$, where $A \in \mathcal{F}(Q)$ and $n \in \mathbb{N}$. Thus $\{x\}$ is a $G_{\delta}$ set in $H(E)$.

Conversely, assume that $\{x\}$ is the intersection of a countable family of neighborhoods of $x$. We may assume that they are of the form $U\left(x ; A, n^{-1}\right)$, where $A \in \mathcal{F}(Q)$ and $n \in \mathbb{N}$, for a certain countable dense set $Q \subset I$ containing 0 and 1. Then, by Proposition 4.20, $Q \supset D_{o}(x)$, hence $\left|D_{o}(x)\right| \leq \aleph_{0}$.

We conclude this section with an existence result.

Proposition 4.22. Let the ordered metric space E satisfy condition $(\mathrm{m})$, and for every $s \in I$ let $F(s)$ be a compact subset of $E$. Assume that for every set $A \in \mathcal{F}(I)$ there is an increasing function $x: A \rightarrow E$ with $x(s) \in F(s)$ for all $s \in A$. Then there exists an increasing function $x: I \rightarrow E$ with $x(s) \in F(s)$ for all $s \in I$.

Proof. Consider the compact space $F=\prod_{s \in I} F(s)$. For every $A \in \mathcal{F}(I)$ let $F_{A}$ denote the set of all $x \in F$ such that $x \mid A$ is increasing. By assumption, each of the sets $F_{A}$ is nonempty, and in view of condition $(\mathrm{m})$ it is clear that $F_{A}$ is compact in $F$. Moreover, the family $\left\{F_{A}: A \in \mathcal{F}(I)\right\}$ is downward directed by inclusion. It follows that this family has a nonempty intersection, which proves the theorem.

5. Increasing functions and the Helly spaces (I). As before, $K$ will stand for a compact space, and $E$ for an ordered metric space. The ideas and facts collected below are, in essence, a somewhat elaborated and generalized version of those already present in [9] (especially in Theorem 1 therein).

It is a simple observation, and yet of key importance for us, that there exist natural one-to-one correspondences between increasing functions $f$ : $I \rightarrow C(K, E)$ and

a) functions $\varphi: I \times K \rightarrow E$ that are increasing in the first variable and continuous in the second variable, as well as

b) continuous maps $F: K \rightarrow H(E)$. 
These correspondences are determined by the equalities

$$
\varphi(s, t)=f(s)(t)=F(t)(s), \quad \text { where } s \in I \text { and } t \in K .
$$

Thus, in particular, $F(t)=p_{t} \circ f$ for every $t \in K$.

The two propositions below gather together a few easy relevant facts.

Proposition 5.1. The map $f \rightarrow F$ described above is a homeomorphism between the spaces $H\left(C_{p}(K, E)\right)$ and $C_{p}(K, H(E))$.

If $L$ is a compact subset of $H(E)$, we define a function $g_{L}: I \rightarrow C(L, E)$ by $g_{L}(s)=\pi_{s}$, where $\pi_{s}: L \rightarrow E$ is the evaluation map (or projection) at the point $s \in I$; thus $\pi_{s}(x)=x(s)$ for $x \in L$. Clearly, $g_{L}$ is an increasing function, and $p_{x} \circ g_{L}=x$ for each $x \in L$. Hence the associated continuous map $G_{L}: L \rightarrow H(E)$ is simply the identity embedding, and $D\left(g_{L}\right)=D(L)$ by equality $(*)$ in Proposition 3.1 .

Note. Functions of the type $g_{L}$ have already been used in the proofs of Propositions 4.2 and 4.9, and of Corollary 4.10.

Proposition 5.2. Let $f: I \rightarrow C(K, E)$ be an increasing function and $F: K \rightarrow H(E)$ the associated continuous map, and let $K_{f}:=F(K)$, a compact subset of $H(E)$. Then

$$
D(f)=D\left(K_{f}\right)=D\left(g_{K_{f}}\right) .
$$

Moreover, the composition operator $c_{f}: g \mapsto g \circ F$ is an order preserving isometric embedding of $C\left(K_{f}, E\right)$ into $C(K, E)$, and $f=c_{f} \circ g_{K_{f}}$.

Proof. The last equality follows from the following chain of relations, where $s \in I$ and $t \in K$, and $\pi_{s}$ is defined on $K_{f}$ :

$$
\begin{aligned}
\left(c_{f} \circ g_{K_{f}}\right)(s)(t) & =c_{f}\left(\pi_{s}\right)(t)=\left(\pi_{s} \circ F\right)(t)=\pi_{s}(F(t))=F(t)(s) \\
& =p_{t}(f(s))=f(s)(t) .
\end{aligned}
$$

Using Proposition 4.9, from the equality $D(f)=D\left(K_{f}\right)$ we now derive the following.

Proposition 5.3. Let $f: I \rightarrow C(K, E)$ be an increasing function. Then $|D(f)| \leq \aleph_{0}$ iff the associated map $F: K \rightarrow H(E)$ takes its values in $H_{0}(E)$ and has a metrizable range.

Corollary 5.4. The space $C(K, E)$ has property $(\lambda)$ iff $E$ has property $(\lambda)$ and every continuous map from $K$ into $H(E)$ has a metrizable range.

This section will be continued as Section 7 below.

6. Metrizing maps on products of compact spaces. In all of this section, $X$ and $X_{j}(j \in J)$ stand for arbitrary compact (Hausdorff) spaces, 
$Z$ for a Hausdorff topological space and, as before, $E$ for an ordered metric space.

Motivated by the last two results of the preceding section, we shall say that a continuous map $f: X \rightarrow Z$ is metrizing if its range, $f(X)$, is a metrizable (compact) subspace of $Z$. The most natural question arising in this context is: For what spaces $X$ and $Z$ every continuous map $f: X \rightarrow Z$ is metrizing? Note that it is certainly so when either $Z$ or $X$ itself is metrizable (for the latter case see $[6, \S 41 . V I$, Th. 3] or [13, 7.6.3]). On the other hand, it may happen that the only metrizing maps are the constant ones, as is the case when the space $X$ is connected and the space $Z$ has no infinite metrizable subspaces (it is so e.g. for $Z=\beta \mathbb{N}$, see $[4,3.6 .15]$ ).

Proposition 6.1. If a compact space $Y$ is a continuous image of the space $X$, and every continuous map from $X$ to $Z$ is metrizing, then so is every continuous map from $Y$ to $Z$.

In view of Proposition 5.3, the situation most interesting for us is when $Z$ is the Helly space $H_{0}(E)$. In this case our results will be much better than in the general case, thanks to the following two properties of $H_{0}(E)$ :

(A) $H_{0}(E)$ is a first-countable space (Corollary 4.6);

(B) A separably metrizable compact subspace of $H_{0}(E)$ is metrizable (Corollary 4.10).

Proposition 6.2. A continuous map $f: X \rightarrow H_{0}(E)$ is metrizing iff the image under $f$ of every separable compact subset of $X$ is metrizable.

Proof. "If": Since for every separable compact set $L \subset f(X)$ one can find a separable compact set $C \subset X$ so that $L=f(C)$, the range of $f$ is separably metrizable. Apply (B).

We may now restate Corollary 3.6 as follows.

COROLlary 6.3. If the compact space $X$ is separably metrizable, then every continuous map $f: X \rightarrow H_{0}(E)$ is metrizing.

We finish this part with the following consequence of Proposition 4.11.

Proposition 6.4. If the compact space $X$ is scattered, then every continuous map $f: X \rightarrow H$ is metrizing; equivalently, the space $C(X)$ has property $(\lambda)$.

We now turn to our basic problem of when a separately metrizing continuous map, defined on a product of compact spaces, is metrizing.

Consider the product space

$$
X_{J}:=\prod_{j \in J} X_{j}
$$


(this sort of notation for product spaces will often be used in what follows). Given a point $u=\left(u_{j}\right) \in X_{J}$ and an index $i \in J$, we denote by $u^{i}$ the homeomorphic embedding of $X_{i}$ into $X_{J}$ that assigns to each point $t \in X_{i}$ the point $\left(x_{j}\right) \in X_{J}$ such that $x_{j}=u_{j}$ for $j \neq i$ and $x_{i}=t$.

Now, a continuous map $f: X_{J} \rightarrow Z$ will be called metrizing in each variable separately, or briefly separately metrizing, if for each $u \in X_{J}$ and $i \in J$ the map $f \circ u^{i}: X_{i} \rightarrow Z$ is metrizing. Thus, in particular, a map $f: X_{1} \times X_{2} \rightarrow Z$ is separately metrizing iff for any $a_{1} \in X_{1}, a_{2} \in X_{2}$ both $f\left(a_{1}, \cdot\right): X_{2} \rightarrow Z$ and $f\left(\cdot, a_{2}\right): X_{1} \rightarrow Z$ have metrizable ranges.

We seek conditions under which a separately metrizing map is metrizing. We start with two auxiliary results. Given a compact space $X$, we denote by $C_{p}(X, Z)$ the space of all continuous maps from $X$ to $Z$ equipped with the topology $\tau_{p}$ of pointwise convergence (the one induced from the product space $Z^{X}$ ).

Proposition 6.5. Let $X_{1}$ and $X_{2}$ be compact spaces, and let $f$ be a continuous map from $X_{1} \times X_{2}$ to $Z$. Then there exist compact spaces $Y_{1}$ and $Y_{2}$, continuous surjective maps $q_{1}: X_{1} \rightarrow Y_{1}$ and $q_{2}: X_{2} \rightarrow Y_{2}$, and a continuous map $g: Y_{1} \times Y_{2} \rightarrow Z$ such that

1) $f=g \circ\left(q_{1}, q_{2}\right)$, i.e., $f\left(x_{1}, x_{2}\right)=g\left(q_{1}\left(x_{1}\right), q_{2}\left(x_{2}\right)\right)$ for all $\left(x_{1}, x_{2}\right)$ in $X_{1} \times X_{2}$

2) both the maps $y_{1} \mapsto g\left(y_{1}, \cdot\right): Y_{1} \rightarrow C_{p}\left(Y_{2}, Z\right)$ and $y_{2} \mapsto g\left(\cdot, y_{2}\right): Y_{2} \rightarrow$ $C_{p}\left(Y_{1}, Z\right)$ are one-to-one.

Proof. Consider the maps $q_{1}: x_{1} \mapsto f\left(x_{1}, \cdot\right)$ from $X_{1}$ to $C_{p}\left(X_{2}, Z\right)$ and $q_{2}: x_{2} \mapsto f\left(\cdot, x_{2}\right)$ from $X_{2}$ to $C_{p}\left(X_{1}, Z\right)$. They are obviously continuous, hence their ranges,

$$
Y_{1}:=\left\{f\left(x_{1}, \cdot\right): x_{1} \in X_{1}\right\} \quad \text { and } \quad Y_{2}:=\left\{f\left(\cdot, x_{2}\right): x_{2} \in X_{2}\right\},
$$

are compact subsets of the spaces $C_{p}\left(X_{2}, Z\right)$ and $C_{p}\left(X_{1}, Z\right)$, respectively.

Next, define a map $g: Y_{1} \times Y_{2} \rightarrow Z$ by setting $g\left(y_{1}, y_{2}\right)=f\left(x_{1}, x_{2}\right)$, where $\left(x_{1}, x_{2}\right)$ is any point in $X_{1} \times X_{2}$ such that $y_{1}=f\left(x_{1}, \cdot\right)$ and $y_{2}=f\left(\cdot, x_{2}\right)$.

This makes sense because if $f\left(x_{1}, \cdot\right)=f\left(x_{1}^{\prime}, \cdot\right)$ and $f\left(\cdot, x_{2}\right)=f\left(\cdot, x_{2}^{\prime}\right)$ for some $\left(x_{1}^{\prime}, x_{2}^{\prime}\right)$, then $f\left(x_{1}, x_{2}\right)=f\left(x_{1}^{\prime}, x_{2}\right)=f\left(x_{1}^{\prime}, x_{2}^{\prime}\right)$.

From the definition of $g$ it is clear that both conditions 1) and 2) are satisfied. Moreover, since $\left(q_{1}, q_{2}\right)$ is a continuous surjective map of $X_{1} \times X_{2}$ onto $Y_{1} \times Y_{2}$ (hence a quotient map) and $f$ is continuous, so is $g$.

Proposition 6.6. Let $F$ be a compact subset of the space $C_{p}(X, Z)$. If the space $X$ is separable and for each $t \in X$ the (compact) set

$$
F(t):=\{f(t): f \in F\} \subset Z
$$

is metrizable, then also $F$ is metrizable. 
Proof. Let $D$ be a countable dense subset of $X$. Then the map $\left.f \mapsto f\right|_{D}$ from $F$ to the product space $\prod_{t \in D} F(t)$, which is compact and metrizable, is continuous and one-to-one, hence it is a homeomorphic embedding. From this the assertion follows immediately.

We are now ready for our basic result on separately metrizing maps.

Theorem 6.7. Let $\left(X_{j}\right)_{j \in J}$ be a finite $(J=\{1, \ldots, n\})$ or infinite $(J=\mathbb{N})$ sequence of compact spaces, all of which, except possibly one, are separable. If a continuous map $f$ from the product space $X_{J}$ to the space $Z$ is separately metrizing, then it is metrizing.

Proof. We may assume that all the spaces $X_{j}$ for $j \geq 2$ are separable.

We start with the case of a finite sequence $X_{1}, \ldots, X_{n}$. We proceed by induction.

First, let $n=2$. In view of Proposition 6.5 we may assume that the continuous maps $q_{1}: x_{1} \mapsto f\left(x_{1}, \cdot\right)$ from $X_{1}$ to $C_{p}\left(X_{2}, Z\right)$ and $q_{2}: x_{2} \mapsto f\left(\cdot, x_{2}\right)$ from $X_{2}$ to $C_{p}\left(X_{1}, Z\right)$ are one-to-one. Then

$$
F:=q_{1}\left(X_{1}\right)=\left\{f\left(x_{1}, \cdot\right): x_{1} \in X_{1}\right\}
$$

is a compact subset of $C_{p}\left(X_{2}, Z\right)$ and, by assumption, each of the sets $F\left(x_{2}\right)=f\left(X_{1}, x_{2}\right) \subset Z$ is metrizable. Hence, by Proposition $6.6, F$ is metrizable, and since $q_{1}$ is a homeomorphism between $X_{1}$ and $F$, also the space $X_{1}$ is metrizable.

Now, as $X_{1}$ is compact and metrizable, hence separable, interchanging the roles of $X_{1}$ and $X_{2}$ in the above argument shows that also the space $X_{2}$ must be metrizable.

Consequently, both the spaces $X_{1}$ and $X_{2}$ are compact and metrizable, and so are $X_{1} \times X_{2}$ and $f\left(X_{1} \times X_{2}\right)$.

Assume now the assertion holds for some $n=k \geq 2$, and consider the case of $n=k+1$ spaces. Fix any $x_{1} \in X_{1}$ and let $g$ denote the continuous map $\left(x_{2}, \ldots, x_{n}\right) \mapsto f\left(x_{1}, x_{2}, \ldots, x_{n}\right)$ from $Y:=X_{2} \times \cdots \times X_{n}$ to $Z$. It is clear that $g$ is separately metrizing. By the induction hypothesis, $g$ has a metrizable range. Now, the space $Y$ is separable, and $f$ viewed as a map from $X_{1} \times Y$ to $Z$ is separately metrizing. By the case $n=2, f$ is metrizing.

Now, let us deal with the case of an infinite sequence $\left(X_{j}\right)$ of spaces. For every $k \in \mathbb{N}$ set

$$
Y_{k}=\prod_{n=1}^{k} X_{n}, \quad Z_{k}=\prod_{n=k+1}^{\infty} X_{n} .
$$

Fix any $k \in \mathbb{N}$. Then for every point $z \in Z_{k}$ the map $y \mapsto f(y, z)$ from $Y_{k}$ to $Z$ is separately metrizing. Therefore, by the first part of the proof, it has a metrizable range. Denote by $f_{k}$ the continuous map from $Y_{k}$ to $C_{p}\left(Z_{k}, Z\right)$ defined by the equality $f_{k}(y)(z)=f(y, z)$. In view of Proposition 6.6, from 
the preceding observation and since the space $Z_{k}$ is separable it follows that $F_{k}=f_{k}\left(Y_{k}\right)$ is a metrizable compact subset of the space $C_{p}\left(Z_{k}, Z\right)$.

Let $F:=\prod_{k \in \mathbb{N}} F_{k}$, a metrizable compact space, and define a continuous map $q: X_{J} \rightarrow F$ as follows:

$$
q(x)=\left(f_{k}\left(x^{k}\right)\right)_{k \in \mathbb{N}},
$$

where $x:=\left(x_{1}, x_{2}, \ldots\right) \in X_{J}$ and $x^{k}:=\left(x_{1}, \ldots, x_{k}\right) \in Y_{k}$.

Let $G:=q\left(X_{J}\right)$, a metrizable and compact subset of $F$, and let $g \in G$. Suppose $g=q(x)=q(y)$ for some $x, y \in X_{J}$. This means that for each $k$ the maps $f_{k}\left(x^{k}\right)=f\left(x_{1}, \ldots, x_{k}, \cdot\right)$ and $f_{k}\left(y^{k}\right)=f\left(y_{1}, \ldots, y_{k}, \cdot\right)$ from $Z_{k}$ to $Z$ coincide. Consequently, $f(x)=f\left(u_{k}\right)$, where $u_{k}=\left(y_{1}, \ldots, y_{k}, x_{k+1}, x_{k+2}, \ldots\right)$. On the other hand, since $u_{k} \rightarrow y$ as $k \rightarrow \infty$, we have $f\left(u_{k}\right) \rightarrow f(y)$ as $k \rightarrow \infty$. Hence $f(x)=f(y)$.

We may therefore define a map $\tilde{f}: G \rightarrow Z$ by setting, for each $g \in G$, $\widetilde{f}(g)=f(x)$, where $x$ is any point in $X$ such that $q(x)=g$. From this definition it is clear that $f=\tilde{f} \circ q$. It follows that $\tilde{f}$ is continuous and that $f\left(X_{J}\right)=\widetilde{f}(G)$ is metrizable.

EXAmple 6.8. Theorem 6.7 fails to hold even in the case of two compact spaces $X_{1}$ and $X_{2}$ if the separability hypothesis is omitted.

To see this, let $K=\{0,1\}^{I}$, where $I=[0,1]$, and note that the map $m: K \times K \rightarrow K$ defined by $m(x, y)=x y$ (pointwise multiplication) is continuous. Next, consider the set $X$ of all elements $x \in K$ such that $x(s)=1$ for at most one point $s \in I$. As is easily seen, $X$ is a nonseparable compact subset of $K$. Now, $f:=m \mid X \times X$ is a continuous map from $X \times X$ onto $X$, and it is separately metrizing because $f(x, X)=f(X, x)=\{0, x\}$ for every $x \in X$. However, $X=f(X \times X)$ is not metrizable.

Note that, in the example above, the function 0 is the only nonisolated point of $X$, hence $X$ is homeomorphic to the one-point compactification of the discrete space $[0,1]$. It is also worth noting that the space $X$ can be viewed as the weak closure in the Banach space $l_{2}(I)$ of the set of its unit vectors $e_{s}(s \in I)$. Thus without the separability assumption Theorem 6.7 fails to hold even if $X_{1}, X_{2}$, and $Z$ are Eberlein compacts.

An analogue of Theorem 6.7 for arbitrarily large families $\left(X_{j}\right)$ of separable compact spaces requires an extra assumption on the space $Z$.

TheOREM 6.9. Let $X_{j}(j \in J)$ be separable compact spaces and assume that each point in the space $Z$ is a $G_{\delta}$ set. If a continuous map $f$ from the product space $X_{J}$ to the space $Z$ is separately metrizing, then it is metrizing.

Proof. By a result due to Corson and Gleason (see [3], [4, 2.7.13]), $f$ depends only on a countable number of coordinates. Thus there exists a countable subset $T$ of $J$ and a continuous map $g: X_{T} \rightarrow Z$ such that 
$f=g \circ p_{T}$, where $p_{T}$ is the natural projection from $X_{J}$ onto $X_{T}$. Clearly, $g$ is separately metrizing and $f\left(X_{J}\right)=g\left(X_{T}\right)$, and to get the assertion it suffices to apply Theorem 6.7.

Corollary 6.10. Let $X_{j}(j \in J)$ be compact spaces and assume that each point in the space $Z$ is a $G_{\delta}$ set. Also, let $f$ be a separately metrizing continuous map from the product space $X_{J}$ to the space $Z$. Then every separable subspace of the range $f\left(X_{J}\right)$ of $f$ is metrizable. In particular, if $f\left(X_{J}\right)$ is separable, then $f$ is metrizing.

Proof. Take any closed separable subspace $W$ of $f\left(X_{J}\right)$. Then there exists a closed separable subspace $Y$ of $X_{J}$ such that $f(Y)=W$. For each $j \in J$, let $Y_{j}$ denote the image of $Y$ under the natural projection of $X_{J}$ onto $X_{j}$; clearly, $Y_{j}$ is a separable compact subspace of $X_{j}$. To finish, apply Theorem 6.9 to the restriction of $f$ to the subspace $Y_{J}:=\prod_{j \in J} Y_{j}$ of $X_{J}$.

In view of Proposition 6.2, the following is an immediate consequence of the preceding corollary and the fact that $H_{0}(E)$ is a first-countable space.

COROLlary 6.11. A separately metrizing continuous map from any product of compact spaces into the Helly space $H_{0}(E)$ is always metrizing.

Corollary 6.12. Let $X_{j}(j \in J)$ be compact spaces, where $|J| \leq 2^{\aleph_{0}}$, at most one of which is nonseparable. Moreover, assume that each point in the space $Z$ is a $G_{\delta}$ set. If a continuous map $f$ from the product space $X_{J}$ to the space $Z$ is separately metrizing, then it is metrizing.

Proof. We only need to consider the case where one of the spaces $X_{j}$, say $X_{j_{0}}$, is nonseparable. Define $X=X_{j_{0}}$ and $Y=\prod_{j \in J \backslash j_{0}} X_{j}$. Then the space $Y$ is separable (by $[4,2.3 .16]$ ), and using Theorem 6.9 it is easily seen that $f$, as a map from $X \times Y$ into $Z$, is separately metrizing. An appeal to Theorem 6.7 finishes the proof.

EXAMPLE 6.13 . Theorem 6.9 becomes false if one omits the assumption that each point in $Z$ is a $G_{\delta}$ set. Let $\left\{I_{j}: j \in J\right\}$ be a partition of $I=[0,1]$ into infinite countable subsets; of course, $|J|=2^{\aleph_{0}}$. For each $j \in J$, let $X_{j}=D^{I_{j}}$, and let $Z=D^{I}$, where $D$ stands for the discrete space $\{0,1\}$. Note that each of the compact spaces $X_{j}$ is metrizable, and that no point in $Z$ is a $G_{\delta}$ set. Consider the map $f$ that assigns to each point $x=\left(x_{j}\right)$ in $X_{J}$ the point $z \in Z$ such that $z \mid I_{j}=x_{j}$ for every $j \in J$. Clearly, $f$ is a homeomorphism between $X_{J}$ and $Z$, and it is separately metrizing. However, $Z=f\left(X_{J}\right)$ is not metrizable.

7. Increasing functions and the Helly spaces (II). In this section, $E$ is an ordered metric space, and $K_{J}$ stands for the product of a family $\left(K_{j}: j \in J\right)$ of compact spaces. For each point $u \in K_{J}$ and index $i \in J$, the 
homeomorphic embedding $u^{i}: K_{i} \rightarrow K_{J}$ is defined as at the beginning of Section 6.

TheOREm 7.1. Let a function $f: I \rightarrow C\left(K_{J}, E\right)$ be increasing. Assume that for every point $u=\left(u_{j}\right) \in K_{J}$ and every index $i \in J$ the function $s \mapsto f(s) \circ u^{i}$ from $I$ to $C\left(K_{i}, E\right)$ has at most countably many discontinuities. Then $f$ itself has at most countably many discontinuities.

Proof. Consider the map $F: K_{J} \rightarrow H(E)$ associated with $f$ (see Section 5). Thus $F(t)(s)=f(s)(t)$ for $t=\left(t_{j}\right) \in K_{J}, s \in I$. Since $F(t)(s)=$ $f(s) \circ t^{i}\left(t_{i}\right)$, we have $|D(F(t))| \leq \aleph_{0}$ so that the range of $F$ is actually contained in $H_{0}(E)$. From the assumptions on $f$ and Proposition 5.3 it now follows that $F: K_{J} \rightarrow H_{0}(E)$ is separately metrizing. To conclude, apply Corollary 6.11 and Proposition 5.3 again.

Theorem 7.2. For every $j \in J$ let $X_{j}$ be a subspace of $C\left(K_{j}, E\right)$ having property $(\lambda)$, and define $X$ to be the subspace of $C\left(K_{J}, E\right)$ consisting of all functions $x$ such that $x \circ u^{i} \in X_{i}$ for all $u \in K_{J}$ and $i \in J$. Then $X$ has property $(\lambda)$.

Proof. Let $f: I \rightarrow X$ be an increasing function. Then each of the increasing functions $s \mapsto f(s) \circ u^{i}$ from $I$ to $X_{i} \subset C\left(K_{i}, E\right)$ has at most countably many discontinuities because $X_{i}$ has property $(\lambda)$. To finish, apply the preceding theorem.

Corollary 7.3. If all the spaces $C\left(K_{j}, E\right)$ have property $(\lambda)$, so does the space $C\left(K_{J}, E\right)$.

Corollary 7.4. Let $K$ be a compact space and $E$ a Banach lattice. If both the spaces $C(K)$ and $E$ have property $(\lambda)$, then also the space $C(K, E)$ has property $(\lambda)$.

Proof. Let $U$ denote the positive part of the closed unit ball in the dual Banach lattice $E^{*}$; equipped with the induced weak* topology, $U$ is a compact space. Then $E$ can be viewed, via a natural order-preserving linear isomorphic embedding, as a subspace of $C(U)$. Hence, likewise, $C(K, E)$ can be viewed as subspace of $C(K, C(U)) \cong C(K \times U)$. To finish, apply Theorem 7.2 with $X_{1}=C(K)$ and $X_{2}=E \subset C(U)$.

\section{References}

[1] S. Banach, Théorie des Opérations Linéaires, Warszawa, 1932; reprinted in: S. Banach, Oeuvres, Vol. II, PWN-Éditions Scientifiques de Pologne, Warszawa, 1979.

[2] L. Drewnowski, Continuity of monotone functions with values in Banach lattices, in: Recent Progress in Functional Analysis (Valencia, 2000), Elsevier, 2001, 185-199.

[3] R. Engelking, On functions defined on Cartesian products, Fund. Math. 59 (1966), 221-231. 
[4] R. Engelking, General Topology, PWN-Polish Sci. Publ., Warszawa, 1977.

[5] J. L. Kelley, General Topology, van Nostrand, New York, 1955.

[6] K. Kuratowski, Topology. I, Academic Press, New York, and PWN-Polish Sci. Publ., Warszawa, 1966.

[7] K. Kuratowski and A. Mostowski, Set Theory, PWN-Polish Sci. Publ., Warszawa, and North-Holland, Amsterdam, 1976.

[8] B. Lavrič, A characterization of Banach lattices with order continuous norm, Rad. Mat. 8 (1992), 37-41.

[9] A. Michalak, On monotonic functions from the unit interval into a Banach space with uncountable sets of points of discontinuity, Studia Math. 155 (2003), 171-182.

[10] - On continuous linear operators on $D(0,1)$ with nonseparable ranges, Comment. Math. (Prace Mat.) 43 (2003), 221-248.

[11] S. Negrepontis, Banach spaces and topology, in: Handbook of Set-Theoretic Topology, K. Kunen and J. E. Vaughan (eds.), Elsevier, 1984, 1045-1142.

[12] W. F. Pfeffer, On some subspaces of the Helly space, Colloq. Math. 35 (1976), 41-46.

[13] Z. Semadeni, Banach Spaces of Continuous Functions, Vol. I, PWN-Polish Sci. Publ., Warszawa, 1971.

Faculty of Mathematics and Computer Science

A. Mickiewicz University

Umultowska 87

61-614 Poznań, Poland

E-mail: drewlech@amu.edu.pl

michalak@amu.edu.pl

Received 16 January 2007 